\title{
Study and Design of Micro Gripper Driven by Electrothermal V-Shaped Actuator
}

\author{
Pham Hong Phuc*, Bui Van Dien, Pham Manh Cuong \\ Hanoi University of Science and Technology, Hanoi, Vietnam \\ "Email: phuc.phamhong@hust.edu.vn
}

\begin{abstract}
This paper presents a design, calculation, simulation and fabrication of micro gripper driven by the electrothermal $V$-shaped actuator. The working principle of the $V$-shaped actuator bases on the thermal expansion of a thin beam when applying a voltage. The advantages of this design are large displacement amplification factor, large driving force, low voltage, simple configuration and control. The maximum displacement of each jaw can be up to $40 \mu \mathrm{m}$ at the calculated voltage of $17.35 \mathrm{~V}$ and low operating frequency. Simulating displacement of the micro gripper in ANSYS multiphysics shows an average voltage deviation of $5.98 \%$ in comparison with calculation at the same displacement. The micro gripper has been fabricated successfully by using MEMS technology and SOI wafer. Measured result confirmed positive features of the system such as large displacement and low driving voltage (i.e. low power consumption). The next step, it can be integrated in micro-robot or in micro assembling systems to clamp and lift the micro/nano samples.
\end{abstract}

Keywords: Micro gripper, V-shaped actuator, Bulk-micromachining, Silicon on insulator (SOI) wafer.

\section{Introduction}

Micro gripper is one of the featured devices of MEMS (Micro - Electro - Mechanical - Systems) technology, consists of capable of handling and manipulating (grasping, grip, or moving) with micrometer accuracy. Some applications of the micro gripper can be mentioned as micro-assembly, microsurgery, biological experiments, materials science, micro-structure research, etc. Specially, the micro gripper is an efficient tool for pick-and-place of individual particles or bio-cells [1]. It can be used as an end-effector in a robotic system, providing an automated operation.

There are various types of micro gripper that have been researched and fabricated. Basing on the driving physics effects, they are categorized as electrostatic, electrothermal, piezoelectric, electromagnetic and shape memory alloys (SMA) [2]. Among these, the electrothermal actuator has some advantages: large driving force, low driving voltage, simple control and fabrication [2,3]. The development of miniaturized actuators became possible with the advances in deep X-ray lithography, LIGA (German acronym for Lithography, electroplating, and molding) and deep reactive ion etching (DRIE) techniques, which allowed fabrication of devices with the required high aspect ratio. Electrothermal actuators require relatively easy fabrication processes, compatible with the standard Integrated Circuits (IC) and MEMS fabrication process. Most of the actuators are

ISSN 2734-9373

https://doi.org/10.51316/jst.150.ssad.2021.31.1.14 Received: January 20, 2021; accepted: May 17, 2021 operated in the standard IC voltage range, therefore, the electrothermal actuators can be easily integrated with the IC devices and implemented in the same fabrication steps. Furthermore, the electrothermal actuators do not depend on electrostatic or magnetic fields while working. Thus, they are suitable for manipulation of biological samples and/or nano material testing devices [4].

According to the beam structure, there are three main types of electrothermal actuators like U-shaped (hot-cold arms), V-shaped (chevron) and Z-shaped designs. The U-shaped actuator generates swing motion due to asymmetric thermal expansion of hot arm and cold arm [5]. V-shaped and Z-shaped actuators produce one-way translational motion when the beams are arranged symmetrically through the shuttle [6]. Comparing with the U-shape, the $\mathrm{V}$-shaped provides the displacement greater at the same voltage [7]. For the same size, the V-shaped creates a larger force and displacement than Z-shaped [8]. Thanks to the simple structure, the devices using V-shaped structure are more stable than U-shaped and Z-shaped actuators. For the V-shaped type, larger displacement can be achieved with a smaller inclined angle of beam. Alternatively, a large number of parallel V-beams can also be used to increase the stiffness, axial stability and the output force when designing a high-performance $\mathrm{V}$-shaped structure.

In this paper, we purpose the design of a new micro gripper driven by V-shaped actuator (consists of 10 couples of symmetric thin beam) with the advantages/improvement of low driving voltage, simple fabrication and control, large motion amplification factor. The micro gripper will be 
fabricated by using MEMS technology and silicon on insulator (SOI) material.

\section{Configuration, Working Principle and Heat Transfer Quation}

\subsection{Configuration and Working Principle}

Figure 1 shows the configuration of micro gripper. The working principle of micro gripper bases on thermal expansion of $\mathrm{V}$-beam. When applying a voltage on two fixed electrodes (1), the V-beams (3) will expand and pushes the shuttle (2) moving in Z-direction. The shuttle (2) is linked with U-shaped claw (4). The ends of the U-shaped claw connect to gripping arm (8) by a revolute joint (6). Others of the gripping arm are connected to a fixed pad by two elastic springs (5). When the shuttle moving forward, U-shaped claw will push the jaw (7) moving close together. When the voltage goes to zero, the temperature of $\mathrm{V}$-beam reduces towards room temperature. The beams will shrink and pull the shuttle backward, the jaw will return by the elastic force of two springs (5).

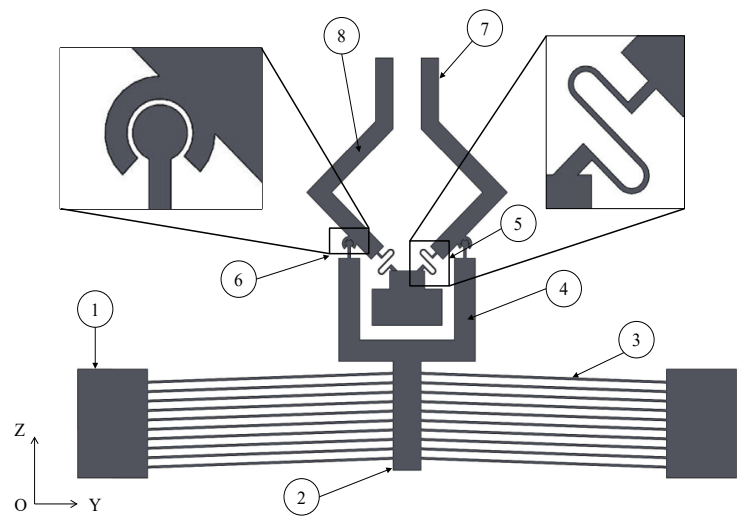

Fig. 1. Configuration of micro gripper

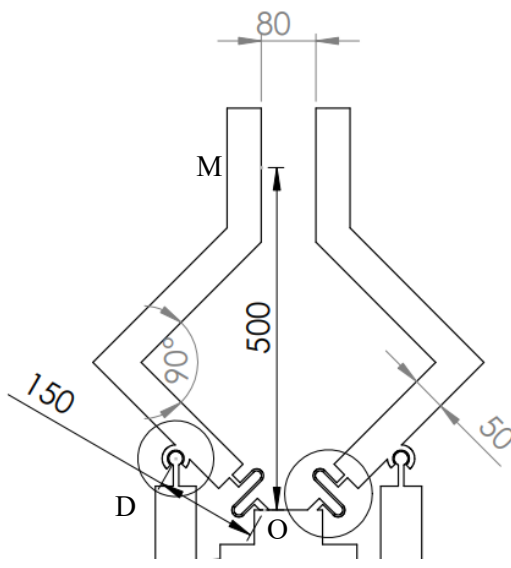

Fig. 2. Dimensions of gripping arm

In this design, the micro gripper has advantages such as: Amplifying the displacement of the shuttle (2) by lever system on gripping arm (8) with amplification factor $k \quad\left(k=\frac{O M}{O D}=\frac{500}{150}=3.33\right)$; Increasing grip capacity due to using elastic springs (5); Reducing concentrated stress at the elastic points by using revolute joint (6). The configuration of gripping arm and jaw is shown in Fig. 2.

The geometrical dimensions of V-shaped actuator are shown in Fig. 3. Where $n$ is the number of beam pairs; $L, h, w$ are the length, height and width of a single beam, respectively; $\theta$ is the inclined angle of $\mathrm{V}$-beam in $\mathrm{Y}$-direction. This $\mathrm{V}$-shaped actuator is based on the design in [9] and integrated to drive micro gripper.

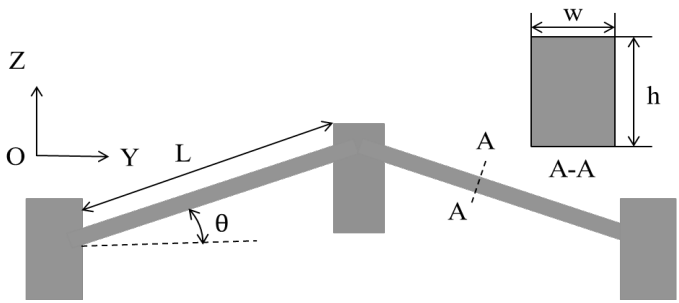

Fig. 3. Configuration of the electrothermal V-shaped actuator

\subsection{The Heat Transfer Model of Electrothermal V-Shaped Actuator}

The analytical equation for heat energy balance in a $\mathrm{V}$-beam is referred from $[10,11]$ :

$$
k_{s} \frac{d^{2} T}{d x^{2}}-\frac{k_{a} S}{h g_{a}}\left(T-T_{o}\right)+\frac{U^{2}}{4 \rho_{o} L^{2}}=0
$$

where, $T$ is the temperature of the beam; $U$ is the driving voltage; $k_{s}$ and $k_{a}$ are the thermal conductivity of single crystal silicon and air; $\rho_{o}$ is the average resistivity of silicon at room temperature $T_{o} ; S$ is the shape factor of beam area section: $S=0.6265\left(\frac{h}{w}\right)+1.1188$ (determined by simulation); $g_{a}$ is a gap between the device layer and substrate (the thickness of $\mathrm{SiO}_{2}$ layer).

By solving the equation (1), a formula for calculating the temperature of a beam element in $x$-direction (along the single beam) is expressed by:

$$
T(x)=T_{o}+\frac{U^{2}}{4 \rho_{o} L^{2}} \frac{h g_{a}}{k_{a} S}+C_{1} e^{\tau x}+C_{2} e^{-\tau x}
$$

where, $C_{1}$ and $C_{2}$ are constants inferred from boundary conditions (at $x=0$ and $x=2 L, T(x)=T_{o}$ ); $\tau$ is the exponential factor defined as follows: 


$$
\tau=\sqrt{\frac{k_{a} S}{k_{S} h g_{a}}}
$$

The total thermal expansion of the single beam can be calculated as:

$$
\begin{gathered}
\Delta L=\int_{0}^{L} \alpha(T) \cdot\left[T(x)-T_{o}\right] \cdot d x= \\
\alpha\left[\frac{U^{2}}{4 \rho_{o} L^{2}} \frac{h g_{a}}{k_{a} S} L+\frac{C_{1}}{\tau}\left(e^{\tau L}-1\right)-\frac{C_{2}}{\tau}\left(e^{-\tau L}-1\right)\right]
\end{gathered}
$$

where $\alpha$ is a thermal expansion coefficient of silicon.

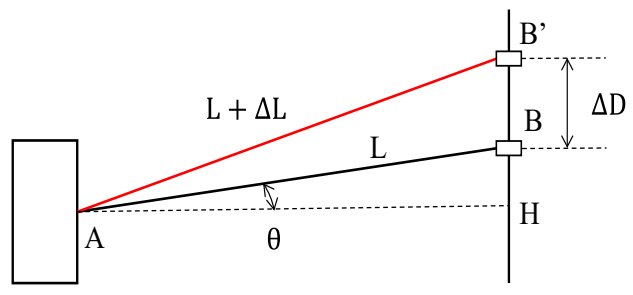

Fig. 4. The displacement of shuttle

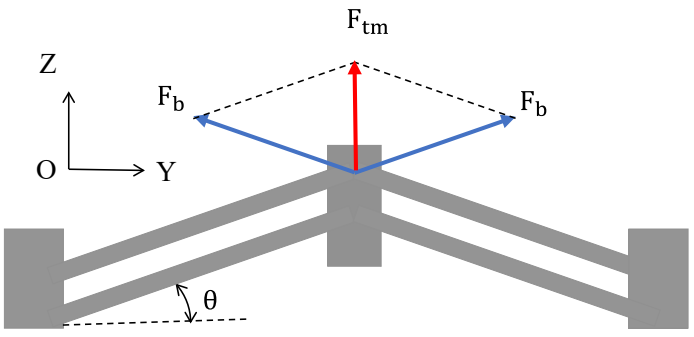

Fig. 5. Thermal expansion force acting on the shuttle

The displacement of shuttle $\Delta D=\mathrm{BB}^{\prime}$ in Z-direction (Fig. 4) can be calculated as:

$$
\begin{gathered}
\Delta D=H B^{\prime}-H B=\sqrt{A B^{\prime 2}-A H^{2}}-H B= \\
\sqrt{(L+\Delta L)^{2}-(L \cos \theta)^{2}}-L \sin \theta
\end{gathered}
$$

The total thermal expansion force $F_{t m}$ of $\mathrm{V}$-beams acting on the shuttle in Z-direction (Fig. 5) is calculated as [9]:

$$
F_{t m}=2 n F_{b} \sin \theta=2 n A E \frac{\Delta L}{L} \sin \theta
$$

where, $F_{b}=A . \sigma=A E \frac{\Delta L}{L}$ is the thermal expansion force generated along the single beam; $A=h . w$ is cross-section area of the beam; $E$ is Young's modulus of silicon; $n$ is the number of V-beam pairs.

\section{Calculation and Simulation of Micro Gripper}

\subsection{Calculation of Jaw Displacement}

The purpose of displacement calculation is to find the relationship between jaw's displacement $\Delta M$ and driving voltage $U$. The movement model of the jaw and gripping arm is shown in Fig. 6. Here, $\Delta M$ is calculated by the following equation:

$$
\begin{aligned}
\Delta M & =k \cdot \Delta K=k \cdot \Delta K_{z} \cdot \cos (\varphi) \\
& =k \cdot\left(\Delta D-g_{K}\right) \cdot \cos (\varphi)
\end{aligned}
$$

where $\Delta K$ is the displacement of the point $\mathrm{D}$ when the gripper arm rotates around the elastic neck of spring (i.e. the point $\mathrm{O}$ in Fig. 6); $\Delta K_{z}$ is the displacement of the point $\mathrm{D}$ on the gripping arm in Z-direction; $g_{K}$ is fabrication gap of the revolute joint (in this design $g_{K}=2 \mu \mathrm{m}$ ).

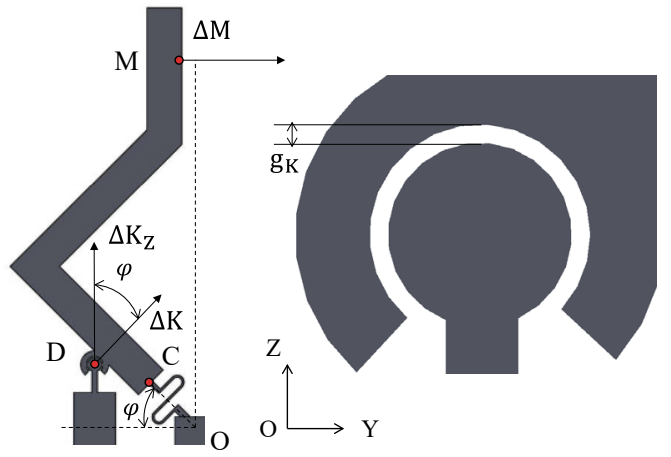

Fig. 6. Configuration of gripping arm and revolute joint

From (5) and (7), the transverse displacement of the jaw is calculated according to the thermal expansion of a single beam as following:

$$
\Delta M=k \cdot\left(\begin{array}{l}
\sqrt{(L+\Delta L)^{2}-(L \cos \theta)^{2}} \\
-L \sin \theta-g_{K}
\end{array}\right) \cdot \cos (\varphi)
$$

From (2), (4), and (8), the relationship between driving voltage $U$ and jaw displacement $\Delta M$ is defined. It is clear that $\Delta M$ is amplified larger than the displacement $\Delta D$ of the shuttle when micro gripper is working.

Geometric dimensions and material properties of micro gripper are given as:

$$
\begin{aligned}
& n=10 ; E=169 \mathrm{GPa} ; \\
& L=700 \mu \mathrm{m} ; h=30 \mu \mathrm{m} ; \\
& w=6 \mu \mathrm{m} ; \alpha=3.33 \times 10^{-6} \mathrm{~K}^{-1} ; \rho_{0}=148 \Omega \mu \mathrm{m} ; \\
& k_{s}=101.25 \times 10^{-6} \mathrm{~W} /(\mu \mathrm{m} . \mathrm{K}) ;
\end{aligned}
$$




$$
\begin{aligned}
& k_{a}=0.0257 \times 10^{-6} \mathrm{~W} /(\mu \mathrm{m} . \mathrm{K}) ; \theta=2^{\circ} ; \\
g_{a}= & 4 \mu \mathrm{m} ; T_{o}=20^{\circ} \mathrm{C} .
\end{aligned}
$$

With the driving voltage changing from 0 to $18 \mathrm{~V}$, the corresponding $\Delta \mathrm{M}$ values are determined and shown in Fig. 10.

\subsection{Calculate the Gripping Force and the Condition for Gripping an Object}

This section establishes the relationship between gripping force $F_{k}$ generated on each jaw and driving voltage $U$ when the jaws grip an object with a specific mass and size. In order to grip the object, it is necessary to calculate the jaw's gripping force acting on the object and find the conditions of the force to be sufficient to hold and lift the object.

Firstly, for moving the gripping arms, the thermal expansion force $F_{t m}$ generated on the shuttle has to be greater than the total hindering forces. It includes the elastic forces $F_{\text {dhd }}$ of the $\mathrm{V}$-beam and $F_{\text {dhlx }}$ of the springs (here, the friction between the gripping arms and substrate is ignored).

$$
F_{t m}>\sum F_{c}=2 F_{d h l x}+F_{\text {dhd }}
$$

The stiffness of each spring can be obtained by simulation (Fig. 9c). The stiffness of V-beam is determined by principle of equivalent displacement in Z-direction. Then, the total stiffness in Z-direction of $n$ beam pairs is calculated as [12]:

$$
k_{t d}=\frac{2 n \cdot E \cdot\left(12 I \cdot \cos ^{2} \theta+A \cdot L^{2} \cdot \sin ^{2} \theta\right)}{L^{3}}
$$

where $I$ is an inertia moment of beam's crosssection area.

With $\Delta D_{\min }=g_{K}=2 \mu m$, the $\mathrm{U}$ - shaped claw can overcome the gap $g_{K}$. Therefore, the minimum driving voltage for passing this gap is:

$$
U_{\text {min }} \geq 4.88 \mathrm{~V}
$$

In order to guarantee that the micro gripper can grip and lift the object with specified dimension, the relationship of voltage and sufficient gripping force is necessary to be examined. Here, the gripped sample is assumed as a silicon cylindrical object with the height $H=60 \mu \mathrm{m}$ and the diameter of $d$.

Fig. 7a shows the diagram of the force acting on gripping arm and gripped object. Here $F_{d}$ is the driving force from the claw acting on the gripping arm; $F_{n}$ is the element force acting on joint $\mathrm{D}$ of the arm in the direction perpendicular to the rotational radius $\mathrm{OD} ; F_{t t}$ is the gripping force at point $\mathrm{M}$ in a jaw generated by voltage $U$.

These forces can be calculated as:

$$
\begin{gathered}
F_{t t}=\frac{F_{n} \cdot O D-F_{d h l x} \cdot O C}{O M} \\
F_{n}=F_{d} \cdot \cos (\varphi)=\left(\frac{F_{t m}-F_{d h d}}{2}\right) \cdot \cos (\varphi)
\end{gathered}
$$

By solving the equation (12), the gripping force $F_{t t}$ ensuring the jaw touching the sample will be determined. But this force is not enough for the gripper to grip and lift the object. We need to add an extra force to the total force $F_{t t}$ so as to overpower object's weight.

(a)
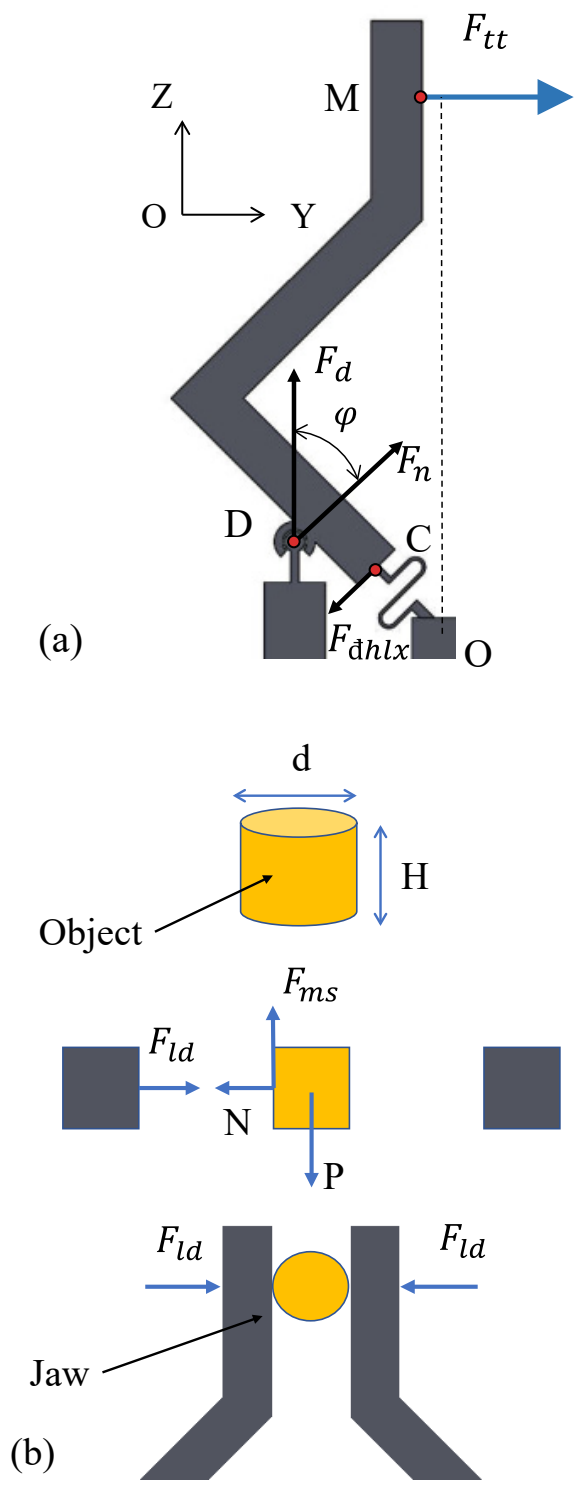

Fig. 7. Force analysis on the gripping arm (a) and on the gripped object (b) 
The friction force $F_{m s}$ exerted by two jaws must be greater than the weight $P$ of the object (Fig. 7b).

$$
\begin{gathered}
2 F_{m s} \geq P ; \\
F_{l d}=N=\frac{P}{2 \cdot f_{m s}}=\frac{m \cdot g}{2 \cdot f_{m s}}=\frac{\pi \cdot d^{2} \cdot H \cdot \rho}{8 \cdot f_{m s}} \cdot g
\end{gathered}
$$

where, $F_{l d}$ is the force that the gripper can lift an object; $f_{m s}=0.3$ is dry friction coefficient of silicon - silicon; $H=60 \mu \mathrm{m}$ is the height of object; $\rho=2330 \mathrm{~kg} / \mathrm{m}^{3} \quad$ is the density of silicon; $g=9.81 \mathrm{~m} / \mathrm{s}^{2}$ is gravitational acceleration; $d$ is diameter of the object.

The minimum gripping force $F_{k}$ that can grip and lift the object is calculated as:

$$
F_{k}=F_{t t}+F_{l d}
$$

From the (12) - (14), the graph of the relation between driving voltage $U$ and object diameter $d$ satisfying the requirement of gripping force $F_{k}$ is shown in Fig. 8.

\subsection{Simulation}

In order to confirm the calculation results and the strength condition of elastic elements, ANSYS software is utilized to simulate displacement, temperature, and maximum stress. Simulation results at $16.6 \mathrm{~V}$ (voltage for the jaws touching the sample) are shown in Fig. 9: The maximum temperature on the V-beam is $T_{m}=545.6^{\circ} \mathrm{C}$ (Fig. 9a); the displacement of shuttle is $\Delta D=18.99 \mu \mathrm{m}$ (Fig. 9b); and the maximum stress of the elastic spring is 98.56 MPa (Fig. 9c).

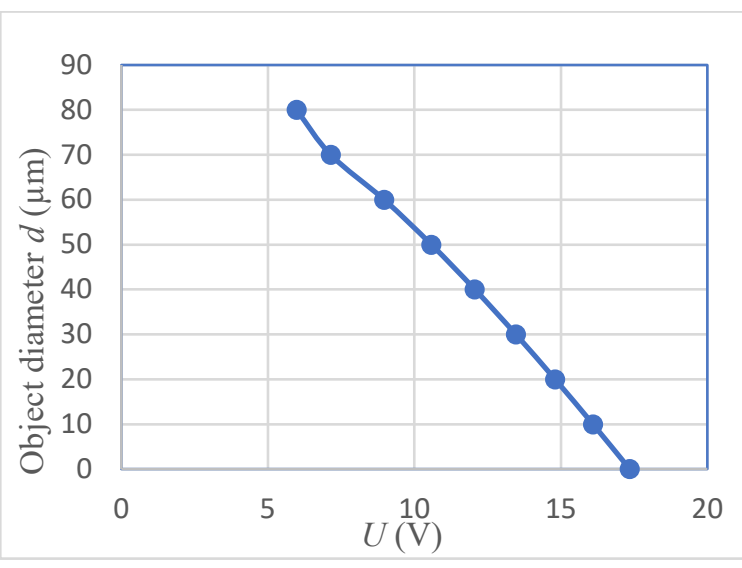

Fig. 8. The relationship between driving voltage and object's diameter
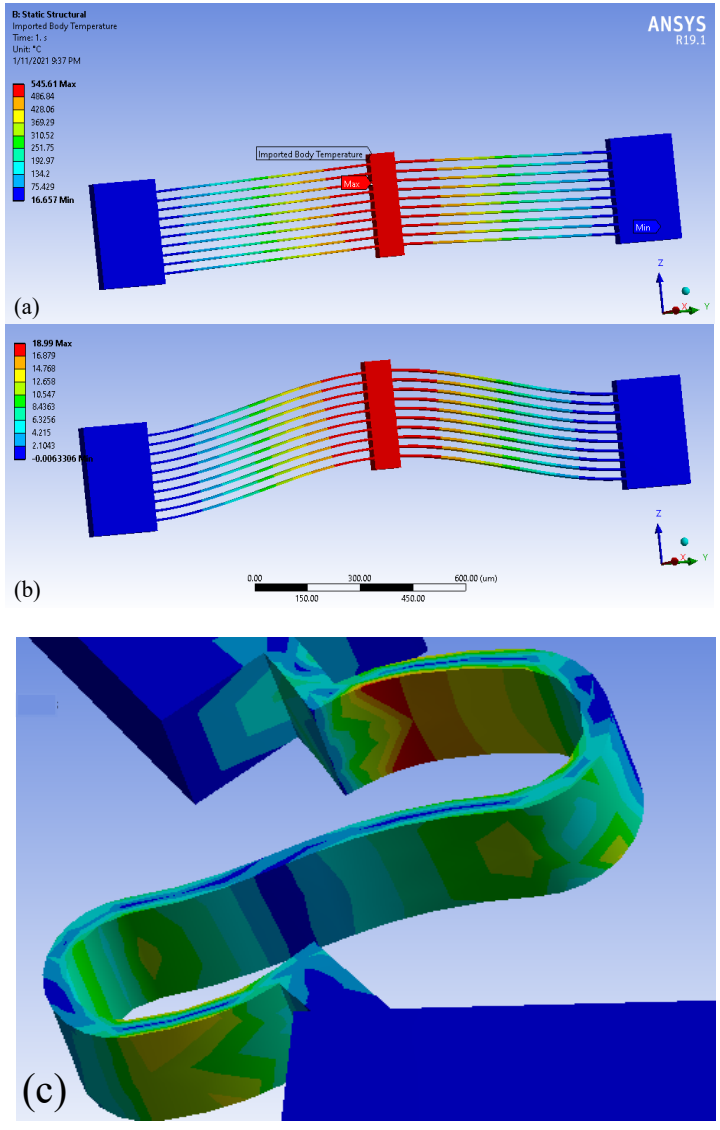

Fig. 9. Simulation result: temperature (a); displacement (b) of the V-beam; and stress of the spring (c) at $U=16.6 \mathrm{~V}$

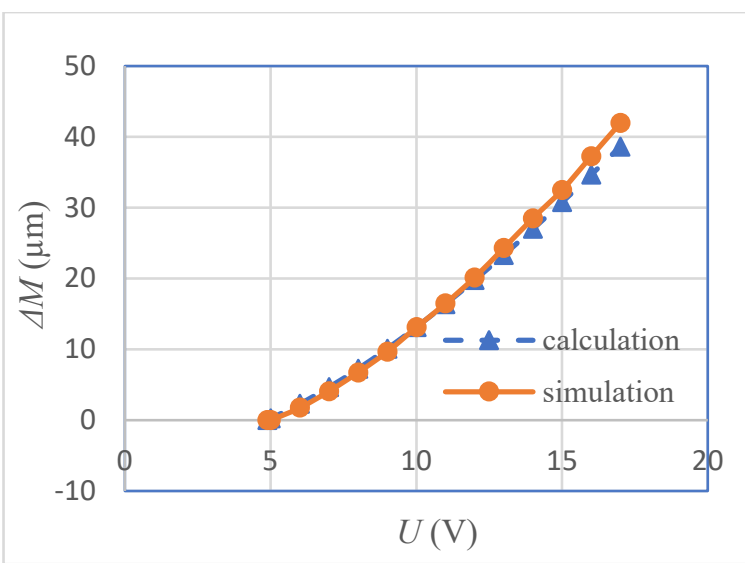

Fig. 10. Calculation and simulation of the jaw's displacement

Fig. 10 shows the calculation results and simulation jaw's displacement $\Delta M$ according to driving voltage $U$. At the same displacement, average deviation of voltage is $5.98 \%$, maximum deviation is $8.6 \%$ at $\Delta M=40 \mu \mathrm{m}$. The reason for deviation can be explained that the analytical method has considered the thermal conductivity coefficients of silicon to be constant when in fact these parameters change and depend remarkably on the temperature. 


\section{Fabrication Process and Result}

\subsection{Fabrication Process}

The micro gripper has been fabricated by bulkmicromachining technology. SOI wafer has three layers, such as silicon layer of $30 \mu \mathrm{m} ; \mathrm{SiO}_{2}$ layer of $4 \mu \mathrm{m}$ and silicon substrate of $450 \mu \mathrm{m}$. The fabrication process consists of four main steps as following [13]: (i) cleaning SOI wafer; (ii) photolithography and developing; (iii) Deep reactive ion etching (DRIE); (iv) removing photoresist and vapor HF etching (see Fig. 11).

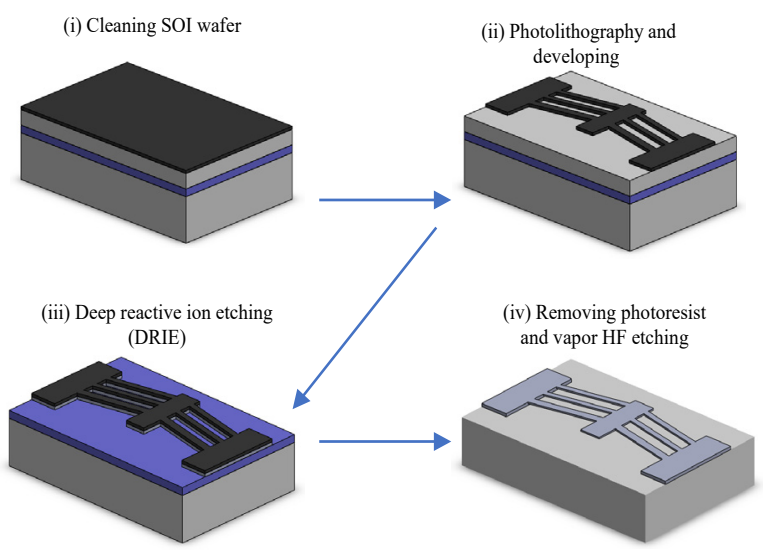

Fig. 11. Fabrication process of the micro gripper using bulk-micromachining technology

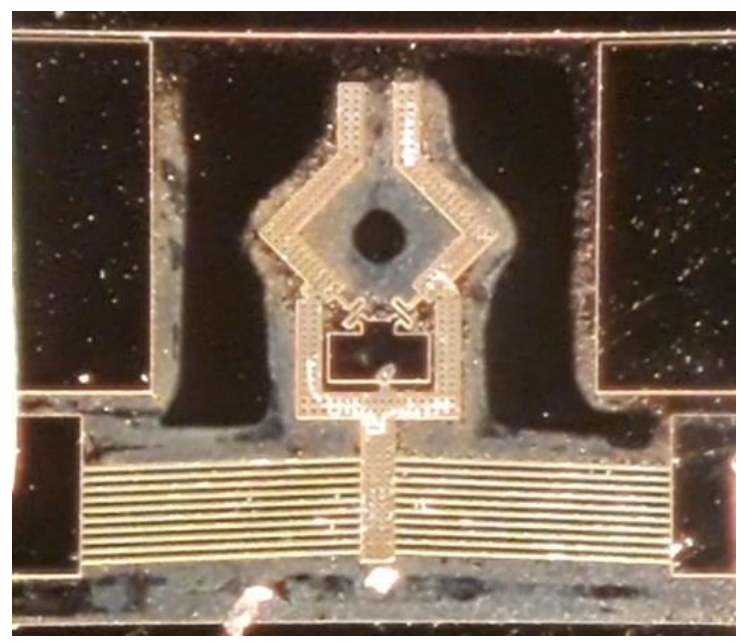

Fig.12. One structure of the gripper after micromachining

First, the photo mask was designed and used for the photolithography process. The gripper structures were transferred to the surface of the SOI wafer after photolithography and developing (step (ii) in Fig. 11). Second, the D-RIE (Deep-Reactive Ion Etching) process was performed to a depth of $30 \mu \mathrm{m}$ to reach the buried oxide layer $\mathrm{SiO}_{2}$ with the etching rate of $1.2 \mu \mathrm{m}$-per-minute (as shown in step (iii)). Then, the SOI wafer was diced to separate each gripper chip with the size of $5 \mathrm{~mm} \times 5 \mathrm{~mm}$. Next, the photoresist layer on the device surface was removed by remover solution, and then the vapor HF etching process was done to etch the $\mathrm{SiO}_{2}$ underneath the device layer and release the movable parts such as V-beams, springs, and gripping arms (step (iv) in Fig. 11). Fig. 12 illustrates one gripper structure after micro fabrication.

After HF etching, the gripper structure is dried at $120{ }^{\circ} \mathrm{C}$ for 10 minutes to further reduce the sticking problem. Then, the device will be evaluated to test operation and determine its characterizations.

The MEMS-based micro gripper has been successfully fabricated by using SOI-MEMS technology in micromachining. The preliminary measurement result has explained that the device can work well at voltages ranging from $2 \mathrm{~V}$ to $10 \mathrm{~V}$.

\subsection{Measurement}

The gripper structure after fabrication has been tested for actual operation and jaw's displacement on the specialized measuring system 4200-SCS supported by Cascade Microtechnology Corporation (see Fig. 13).

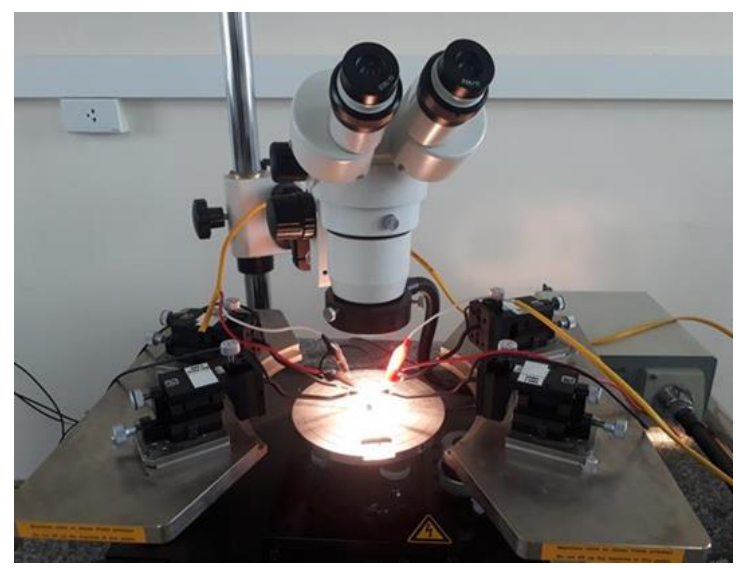

Fig. 13. Microscopy measurement system with 4 probes 4200-SCS

To reduce resistance of $\mathrm{V}$-beams (i.e. reduce applying voltage), the micro gripper structure after fabrication is sputtered by a thin layer of platinum (about 50nm-thick) onto the surface for the better electrical conductivity [12].

In other words, at room condition, there is always a thin $\mathrm{SiO}_{2}$ layer (only some nanometers) covering the top surface of the $\mathrm{V}$-beam layer before sputtering step. This $\mathrm{SiO}_{2}$ membrane has function as an insulation layer. Therefore, after sputtering the resistance of silicon single $\mathrm{V}$-beam and the resistance of platinum layer on its top will create a couple of resistance in parallel and help to reduce evidently the equivalent resistance $R$ of the $\mathrm{V}$-beam system. This 
step is performed by using cathode sputtering system in the clean room located in ITIMS (International Training Institute for Material Science) and helps to reduce a real applying voltage while comparing to simulation and calculation.
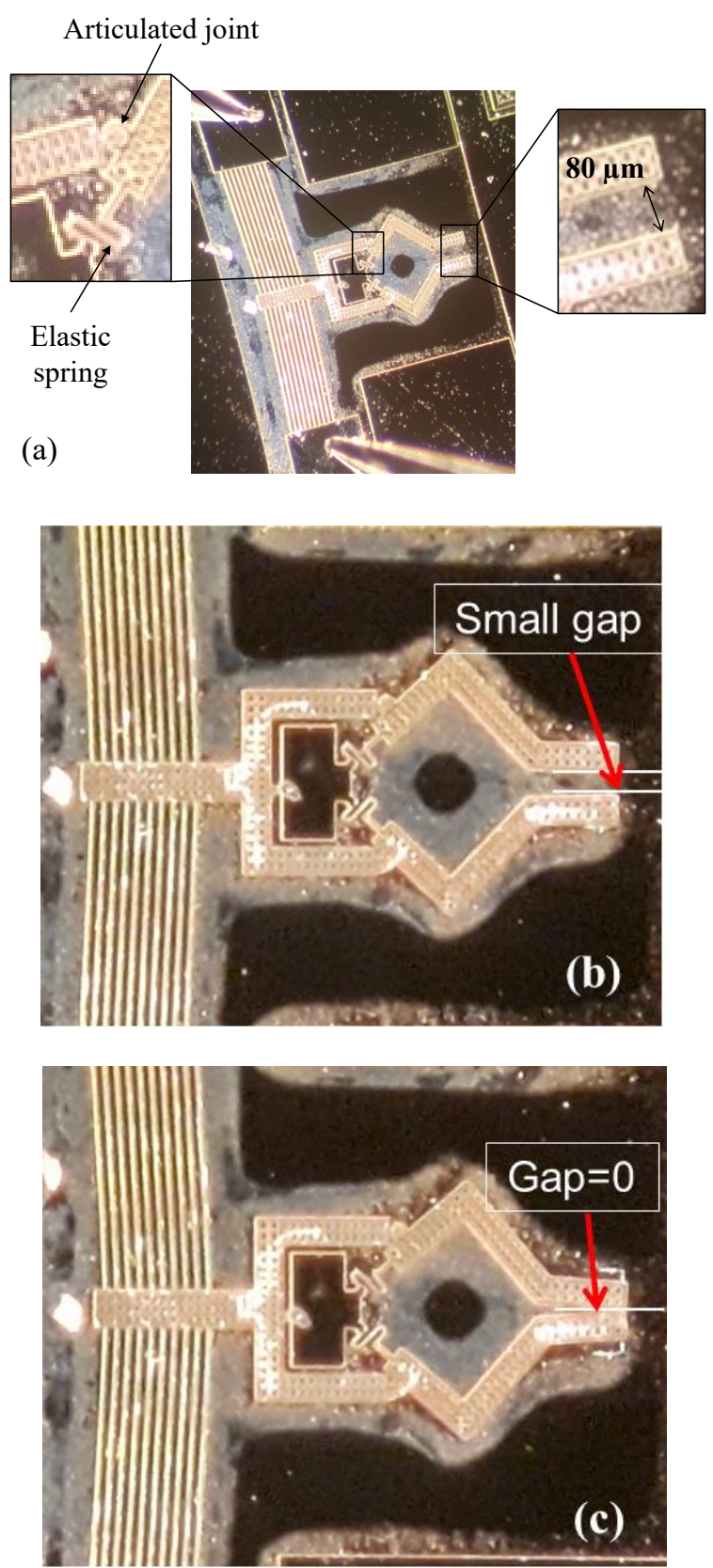

Fig. 14. Operation of micro gripper at the various voltages $U=0 \mathrm{~V}(\mathbf{a}) ; U=6 \mathrm{~V}(\mathbf{b})$; and $U=10 \mathrm{~V}(\mathbf{c})$

Preliminary evaluation of the sputtered-gripper system showed that: At the driving voltage $U=6 \mathrm{~V}$, two jaws move closely (Fig. 14b) and touch each other if $U=10 \mathrm{~V}$ (Fig. 14c), i.e. each jaw can move a stroke of $40 \mu \mathrm{m}$ (the initial distance between two jaws is $80 \mu \mathrm{m}$ as shown in Fig. 14a). It confirms that the operation of the micro gripper matches to design as well as reduces driving voltage (i.e. reduces power consumption) of the device after applying platinum sputtering. The detailed measurement and evaluation of the jaw's displacement at different driving voltages will be continued.

\section{Conclusion}

The paper presented the design, calculation, simulation, and fabrication of the electrothermal micro gripper with the advantages like simple fabrication, large amplification coefficient, low driving voltage and acceptable concentrated stress by using revolute joints. The temperature distribution, displacement and stress of elastic components were examined by ANSYS simulation. The results are relatively matching with theoretical calculations.

The achievements of trial fabrication have exposed the great ability and applications of the device in the future, as well as provide a grasp of the actuation methodology, design, fabrication, and the related performance in cell manipulation, micro assembly, and mechanical testing of micro/nano materials (carbon nano tubes or nano wire), etc.

Remarkable contributions of this design are simple configuration, larger gripping stroke, lower concentrated stress and easy fabrication with only one mask. Besides, low power consumption is valuable improvement of sputtered-device in comparison with previous counterpart. Hence, it allows easily development, integration and application in micro robot, micro assembling or micro conveying systems with flexible gripping/lifting capabilities.

\section{Acknowledgments}

The authors would like to send a thankfulness to Dr. Nguyen Tien Dung for his support in the experiment and measurement.

\section{References}

[1] Ai, W.J. and Xu, Q.S. Overview of flexure-based compliant micro-grippers. Advances in Robotic Research, vol. 1, no. 1, pp. 1-19, 2014. http://dx.doi.org/10.12989/arr.2014.1.1.001

[2] $\mathrm{S}$. Yang and $\mathrm{Q}$. Xu, A review on actuation and sensing techniques for MEMS-based microgrippers, Journal of Micro-Bio Robotics, vol. 13, pp. 1-14, 2017. https://doi.org/10.1007/s12213-017-0098-2.

[3] A. Potekhina and C. Wang, Review of Electrothermal Actuators and Applications, Actuators, 8(4), 2019. https://doi.org/10.3390/act8040069.

[4] H. D. Espinosa, Y. Zhu, and N. Moldovan, Design and operation of a MEMS-based material testing system for nanomechanical characterization, Journal of Microelectromechanical Systems, vol. 16, no. 5. pp. 1219-1231, 2007. https://doi.org/10.1109/JMEMS.2007.905739

[5] J. Varona, M. Tecpoyotl-Torres and A. A. Hamoui, Modeling of MEMS thermal actuation with external heat source, Proceeding of Electronics, Robotics and 
Automotive Mechanics Conference - IEEE, 2007. https://ieeexplore.ieee.org/document/4367751. https://doi.org/10.1109/CERMA.2007.4367751

[6] Z. Zhang, Y. Yu, . X. Liu and X. Zhang, Dynamic modelling and analysis of $\mathrm{V}$ - and Z-shaped electrothermal microactuators, Microsyst Technol., vol. 23, pp. 3775-3789, 2017. https://doi.org/10.1007/s00542-016-3180-0.

[7] J. K. Luo, A. J. Flewitt, S. M. Spearing, N. A. Fleck and W. I. Milne, Comparison of microtweezers based on three lateral thermal actuator configurations, J. Micromech. Microeng, vol. 15, pp. 1294-1302, 2005. https://doi.org/10.1088/0960-1317/15/6/022.

[8] Z. Zhang, Y. Yu, X. Liu and X. Zhang, A comparison model of V- and Z-shaped electrothermal microactuators, International Conference on Mechatronics and Automation - IEEE, pp. 1025-1030, 2015. https://ieeexplore.ieee.org/document/7237626. https://doi.org/10.1109/ICMA.2015.7237626

[9] T. K. Hoang, T. D. Nguyen and P. H. Pham*, Impact of design parameters on working stability of the electrothermal V-shaped actuator, Microsyst Technol., vol. 26, pp. 1479-1487, 2020.

https://doi.org/10.1007/s00542-019-04682-y.

[10] Q. Ji and K. L. Scott, First Order Modeling of Thermal Actuators in SUGAR, Department of Electrical Engineering and Computer Sciences, University of California, Berkeley. Technical note. 2002.

https://people.eecs.berkeley.edu/ pister/245/project/Ji Scott.pdf

[11] T. D. Nguyen, H. P. Pham and Q. Đ. Nguyen, Modification and simulation of electrothermal micromotor, The 3rd National Conference on Control and Automation-VCCA, 2015. (Vietnamese).

[12] T. D. Nguyen, T. K. Hoang and P. H. Pham*, Larger displacement of silicon electrothermal V-shaped actuator using surface sputtering process, Microsyst Technol., vol. 27, no. 5, pp. 1985-1991, 2021. https://doi.org/10.1007/s00542-020-04985-5.

[13] Pham Hong Phuc, Nguyen Tien Dung, Hoang Trung Kien, "Design and Fabrication of the micro actuators based on MEMS-Technique", Science and Technics Publishing House - 2020 (Book in Vietnamese). 